\title{
Reporting di sostenibilità degli operatori logistici in Europa: analisi degli indicatori*
}

\author{
di Enrico Massaroni, Alessandra Cozzolino, Mario Calabrese, \\ Ewa Wankowicz, Maura Fiore
}

Obiettivi: L'affermazione di una nuova concezione del valore, basata sulle qualità e non sull'insostenibile aumento delle quantità di merci e consumi, induce gli operatori logistici alla ricerca di azioni che possano essere nel contempo economicamente efficienti ed efficaci, ecologicamente sopportabili e socialmente giuste. Il presente lavoro ha l'obiettivo di investigare e identificare le azioni sostenibili-economiche, ambientali e sociali - intraprese dagli operatori logistici.

Metodologia: Il quadro teorico è stato costruito attraverso lo studio della letteratura accademica nazionale e internazionale. La parte empirica è stata sviluppata attraverso l'analisi dei più recenti report di sostenibilità degli operatori logistici operanti in Europa, redatti secondo gli standard della Global Reporting Initiative.

Risultati: Gli operatori logistici possono contribuire in modo significativo al cambiamento paradigmatico in atto, facilitando e favorendo, in qualità di fornitori di servizi, il raggiungimento della sostenibilità della supply chain.

Limiti della ricerca: Il presente lavoro costituisce la fase preliminare di un più approfondito progetto di ricerca che indaghi le iniziative di sostenibilità degli operatori logistici a livello mondiale e che consideri un arco temporale di osservazione utile a tracciare il trend in atto.

Implicazioni pratiche: Sia l'accademia che il mondo professionale possono trovare utile l'analisi della letteratura sul tema e i primi risultati ottenuti, in quanto sono posti in evidenzia i principali tratti di sostenibilità degli operatori logistici che già hanno intrapreso il percorso verso la sostenibilità.

Originalità del lavoro: Gli studi della letteratura esistente sugli operatori logistici e la sostenibilità si basano quasi esclusivamente sulla sostenibilità ambientale e l'ecoefficienza, mentre manca un approccio sistemico capace di superare i problemi odierni mediante la valorizzazione della multidimensionalità. Il lavoro contribuisce a colmare questo gap.

Parole chiave: gestione sostenibile della supply chain, operatori dei servizi logistici, reporting di sostenibilità.

* Pur essendo frutto di un lavoro comune, i paragrafi sono attribuiti nel modo seguente: paragrafi 1 e 6 a Enrico Massaroni, 4.2 a Mario Calabrese, 2.2.2 e 5 a Alessandra Cozzolino, 2.1, 2.2.3, 3, 4.1 a Ewa Wankowicz, 2.2.1 a Maura Fiore.

Economia e diritto del terziario n. 2, 2016 
Purposes of the paper: the development of a new concept of value, based on quality and not on unsustainable increase in quantity of goods and consumption, leds logistics providers to the search of actions that would be cost-efficient and effective at the same time, ecologically sustainable and socially fair. This paper wants to investigate and identify sustainable actions - economic, environmental and social - undertaken by logistics providers.

Methodology: the theoretical framework has been realised through the study of the academic literature, both nationally and internationally. The empirical section has been created through analysis of the latest sustainability reports of logistics providers operating in Europe, achieved in accordance with the standards of the Global Reporting Initiative.

Findings: logistics providers may contribute significantly to the paradigm shift now in progress, by facilitating and encouraging, as service providers, the achievement of sustainability in supply chain management.

Research Limits: this work is the preliminary stage of a more detailed research project that requires the sustainability initiatives about worldwide logistics providers and considers a period of observation useful to trace the trends in place.

Practical implications: Both the Academy and the business world can find useful the analysis of the literature on the subject and the first results gained, as the main features of sustainability of logistics providers, which have already embarked on the path towards sustainability, are highlighted.

Originality of the paper: the studies of the existing literature on logistics providers and sustainability are based almost exclusively on environmental sustainability and the eco-efficiency, while a systemic approach able to overcome problems through the enhancement of multidimensionality lacks. The work helps to fill this gap.

Keywords: sustainable supply chain management, logistics service providers, sustainability reporting

JEL Codes: M15IT.

\section{Introduzione}

L'estensione globale delle supply chain ha contribuito alla loro fragilità e frequentemente alla mancata resilienza. In parte questa difficoltà potrebbe essere colmata attraverso la collaborazione tra i diversi attori che partecipano alla creazione di un prodotto o servizio. La necessità di collaborare, sia a monte che a valle, risulta essere cruciale in alcuni casi, in particolare quando le imprese sono chiamate a porre una maggiore attenzione alle problematiche della sostenibilità. Sempre più spesso, la complessa problematica di gestione sostenibile della supply chain (SSCM) coinvolge le imprese di varie dimensioni provenienti da diversi settori (Seuring, 2013). Per la buona riuscita di tale gestione è importante non solo la loro adozione da una singola impresa, ma anche la condivisione delle best pratices con altri attori della catena di distribuzione (Govindan et al., 2013; Van Hoof e Thiell, 2014).

Nell'ambito di questo tema il ruolo degli operatori dei servizi logistici (LSP) può risultare fortemente significativo se considerati alcuni elementi caratterizzanti tali rapporti di fornitura. In particolare, il modus operandi 
dei LSP può determinare il successo della supply chain nel diventare sostenibile, specialmente nell'introduzione e sviluppo di pratiche a favore della sostenibilità. In questo capitolo/paragrafo verrano illustrate alcune iniziative sostenibili introdotte con successo dagli operatori dei servizi logistici operanti in Europa.

\section{Quadro teorico di riferimento}

\subsection{La gestione sostenibile della supply chain}

Per tracciare il quadro di riferimento e per presentare un'evoluzione temporale del concetto del SSCM, nella tabella 1 sono riportate alcune sue definizioni. Tale presentazione ha un duplice obiettivo: cogliere gli aspetti essenziali del SSCM attraverso l'evidenziazione degli elementi caratterizzanti; mettere in evidenza l'ordine con il quale le dimensioni della sostenibilità vengono esplicitate.

Nei lavori di Ciliberti et al. (2008) e Font et al. (2008) il SSCM è presentato come un'integrazione del supply chain management e della sostenibilità. Carter e Rogers (2008), introducono l'elemento del coordinamento dei processi inter-organizzativi con lo scopo di ottenere un miglioramento della performance economica, sia dell'impresa che della supply chain. Seuring e Muller (2008) pongono una maggiore attenzione sulla cooperazione tra le imprese a favore della sostenibilità.

Un importante contributo agli studi sulla supply chain sostenibile viene dato da Ahi e Searcy (2013), i quali esplicitano chiaramente l'aspetto della volontarietà d'impresa nel raggiungimento della sostenibilità, mettendo in evidenza come il coordinamento delle supply chain in ottica sostenibile, possa costituire un punto di partenza verso la profittabilità, competitività e la resilienza nel breve e lungo termine.

Per poter far ciò, è richiesto alle imprese un maggior grado di proattività nella creazione del valore in modo sostenibile, in quanto esso costituisce un obiettivo strategico (Closs et al., 2011; Giunipero et al., 2012). Nonostante allo stato attuale sia carente una definizione del SSCM condivisa dal mondo della ricerca, rimane indubbia la necessità di una considerazione contemporanea delle tre dimensioni della sostenibilità nella gestione della supply chain, affinché essa diventi "veramente sostenibile" (Ahi e Searcy, 2013).

La maggior parte delle definizioni esistenti in letteratura evidenzia, al primo posto, l'importanza della dimensione economica, risultato che, confermando l'affermazione "senza successo economico, non ci sarebbero le supply chain" (Wolf e Seuring, 2010, p. 90), non stupisce considerata la matrice economica-manageriale degli articoli scientifici delle riviste che li ospitano. Risulta più stimolante osservare anche il posizionamento di altri componenti. Il soddisfacimento degli interessi ambientali si trova frequentemente al secondo posto, prevalentemente dopo la performance economica. La sostenibilità ambientale è ricondotta ad 


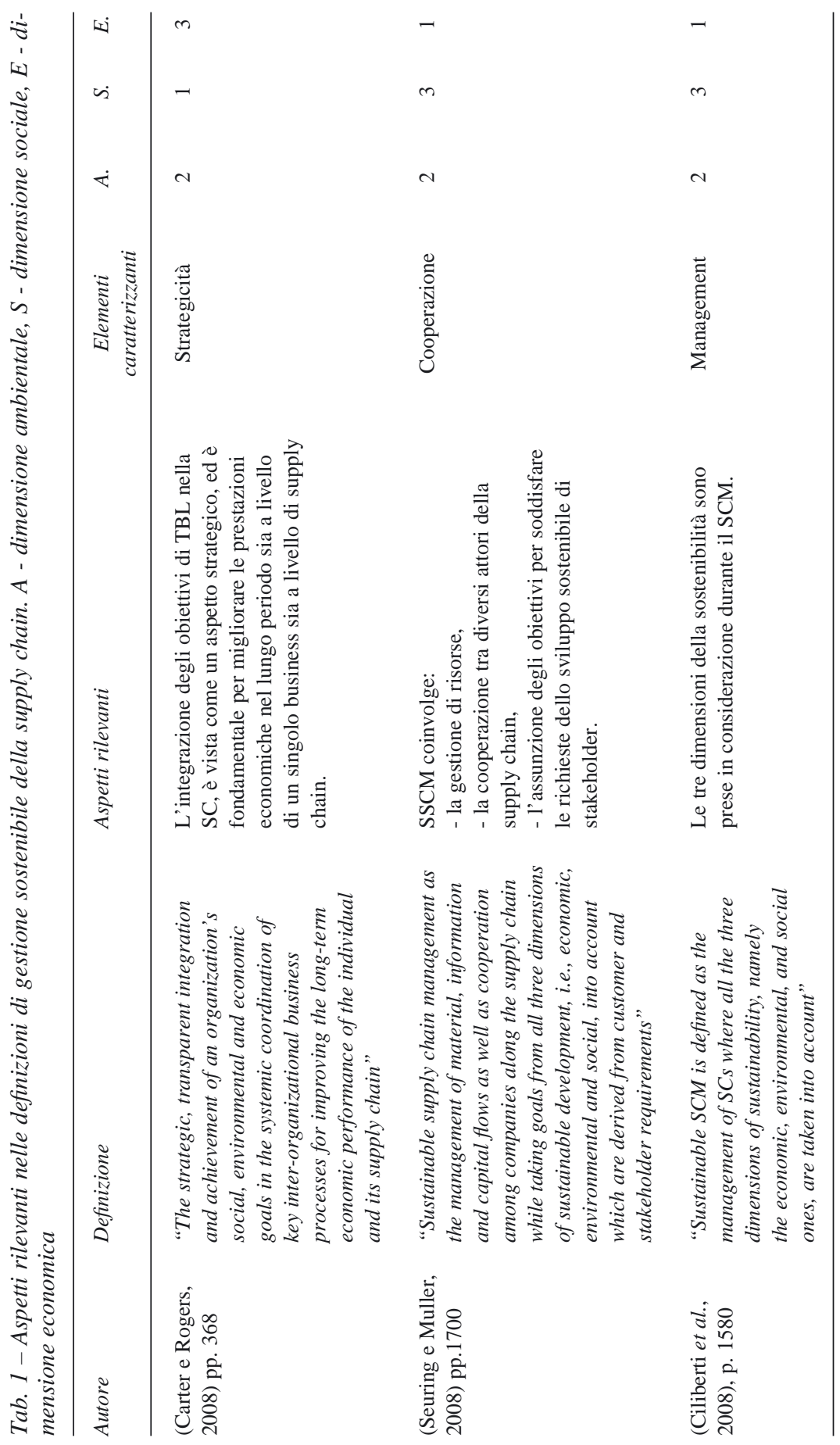




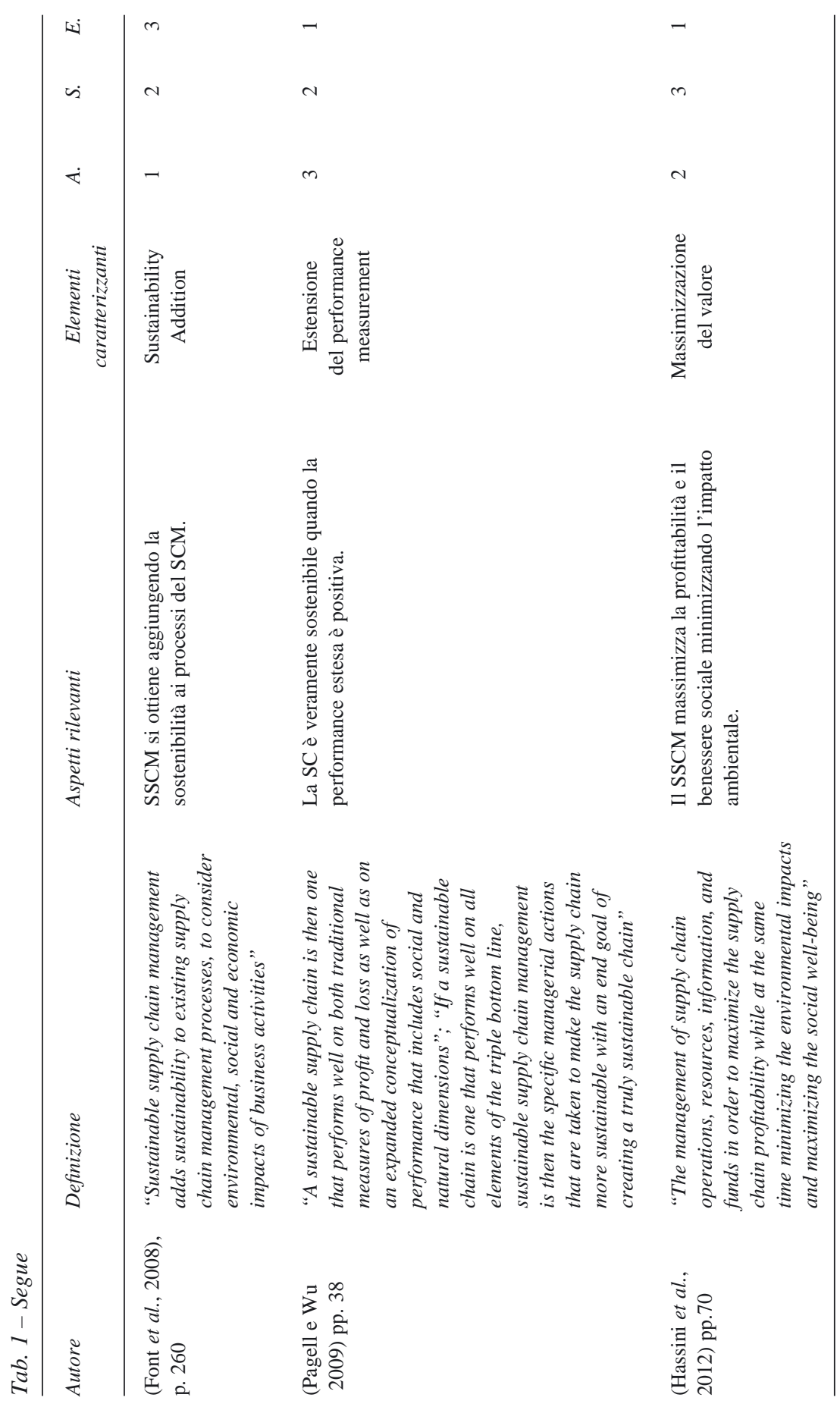

Copyright $($ FrancoAngeli

N.B: Copia ad uso personale. È vietata la riproduzione (totale o parziale) dell'opera con qualsiasi mezzo effettuata e la sua messa a disposizione di terzi, sia in forma gratuita sia a pagamento. 


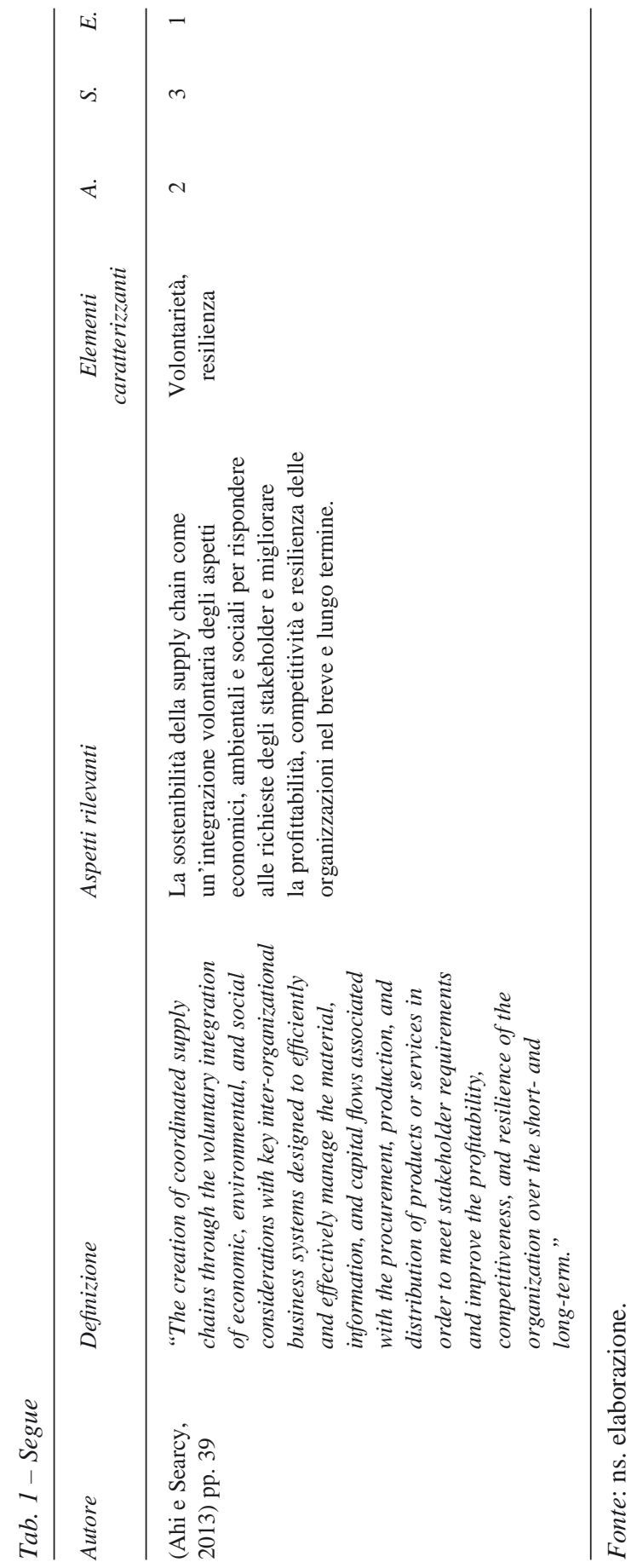
mezzo effettuata e la sua messa a disposizione di terzi, sia in forma gratuita sia a pagamento. 
aspetti di exploitation delle risorse naturali, l'inquinamento atmosferico, il carbon footprint.

L'aspetto sociale, derivante dall'attività economica può impattare sia sull'individuo sia sulla società nel suo complesso (Winter e Knemeyer, 2013), viene menzionato come primo in classifica solo da Carter e Rogers (2008). Esso si riferisce al rispetto dei diritti umani, gestione delle risorse umane attraverso il riconoscimento delle capacità ed abilità individuali, sicurezza sul posto di lavoro, la responsabilità sociale d'impresa (Winter e Knemeyer, 2013; Hassini et al., 2012).

Fino ad oggi la letteratura ha trattato sporadicamente la tridimensionalità della sostenibilità (Seuring e Muller, 2008; Lee e Wu, 2014), ma " $a c$ counting for sustainability aspects of logistics presents a major challenge for the future" (Burritt e Schaltegger, 2014, p. 333).

La prospettiva delle imprese di logistica può risultare in questo senso interessante. L'espansione delle pratiche di outsourcing logistico infatti contribuisce ad attribuire agli operatori logistici un ruolo rilevante nella diffusione della sostenibilità (Facanha e Horvath, 2005; Anttonen et al., 2013; Rossi et al., 2013). L'assegnazione di questo ambizioso "mandato morale" (Hart e Milstein, 2003) deriva dalla responsabilità che hanno i LSP nella gestione delle merci lungo l'intera supply chain; per questo motivo essi godono di un enorme potenziale nella ricerca di sostenibilità (Dey et al., 2011).

\subsection{Operatori logistici e la sostenibilità}

\subsubsection{Analisi sistematica della letteratura}

Al fine di verificare la letteratura esistente sul tema degli operatori logistici e la sostenibilità della supply chain è stata eseguita una ricerca a livello internazionale sulle seguenti banche dati: Ebsco Host (all'interno della quale sono state selezionate in particolare le banche dati Business Complete Source, Eco Lit, Green File), Science Direct e Scopus. A livello nazionale sono state consultate le riviste: Sinergie, Finanza marketing e produzione, Economia e management. Nelle tabelle 2 e 3 sono riportate le parole chiave utilizzate per la ricerca ed i risultati ottenuti, considerando i soli articoli relativi alle pubblicazioni accademiche. Si è riportato il primo anno di pubblicazione con lo scopo di poter prendere conoscenza dell'anno in cui il tema ha iniziato ad essere trattato ed avere dunque un'idea, sulla base della numerosità degli articoli, dell'importanza dello stesso nella letteratura accademica. Dalle tabelle emerge che le pubblicazioni degli articoli riguardanti i temi di ricerca si concentrano soprattutto nell' arco temporale tra il 2010 ad oggi: da questa evidenza deriva la scelta di analizzare più approfonditamente le pubblicazioni di questo specifico periodo. 


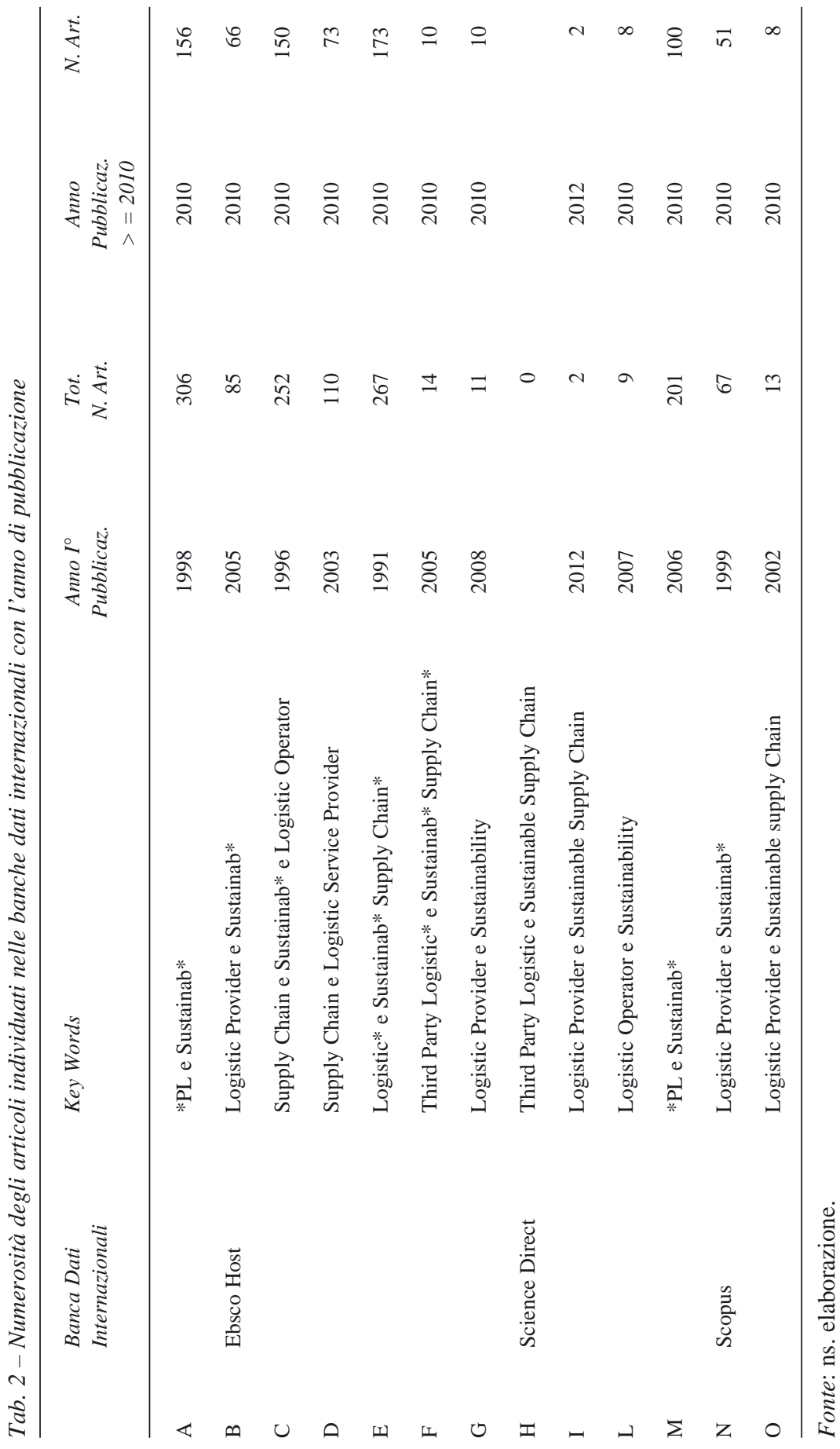

Copyright $($ FrancoAngeli

N.B: Copia ad uso personale. È vietata la riproduzione (totale o parziale) dell'opera con qualsiasi mezzo effettuata e la sua messa a disposizione di terzi, sia in forma gratuita sia a pagamento. 


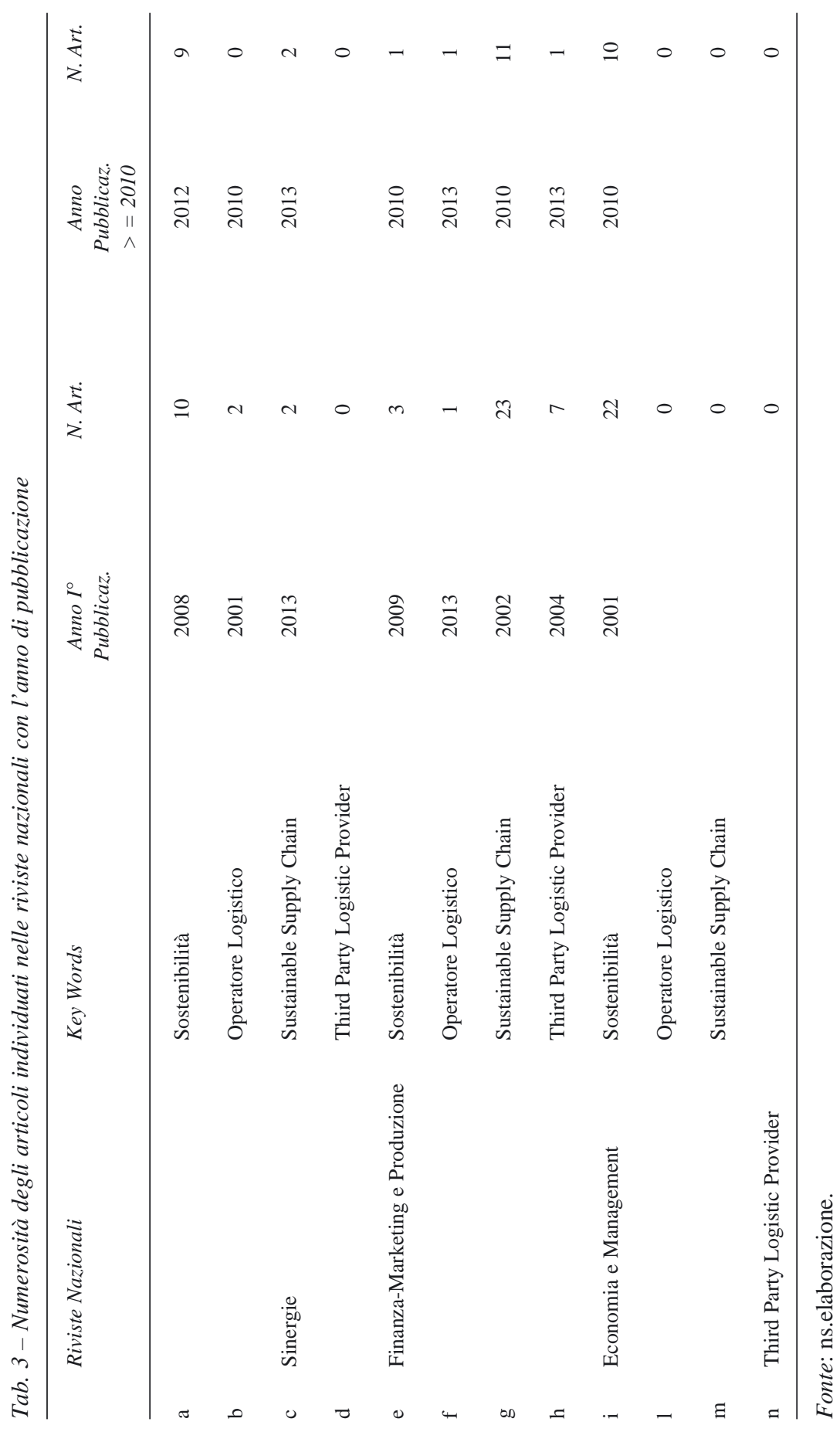

Copyright (C) FrancoAngeli

N.B: Copia ad uso personale. È vietata la riproduzione (totale o parziale) dell'opera con qualsiasi mezzo effettuata e la sua messa a disposizione di terzi, sia in forma gratuita sia a pagamento. 
Da un'accurata lettura degli abstract sono stati selezionati gli articoli maggiormente interessanti: si è proceduto, successivamente, ad una ulteriore scrematura considerando la congruità dell'articolo col tema di ricerca. I lavori scelti sono riportati in tabella 4 .

Tab. 4 - Considerazioni rilevanti emersi dalla literature review sul tema di ricerca

\begin{tabular}{lll}
\hline Autore/Anno & Obiettivo di ricerca & Considerazioni rilevanti \\
\hline Lieb e Lieb, 2010 & $\begin{array}{l}\text { Esaminare le iniziative di } \\
\text { sostenibilità attuate da 3PL e } \\
\text { l'impatto che queste iniziative } \\
\text { hanno sui propri clienti. }\end{array}$ & $\begin{array}{l}\text { Nonostante la crisi economica } \\
\text { dell'ultimo decennio, numerosi LSP } \\
\text { hanno investito in progetti sostenibili } \\
\text { che hanno avuto un impatto positivo } \\
\text { sulla redditività. }\end{array}$
\end{tabular}

Wolf e Seuring, 2010

Analizzare se le questioni ambientali rientrano tra i criteri di selezione nella scelta degli operatori logistici.

Colicchia et al., $2013 \quad$ I LSP possono contribuire a ridurre il peso dell'inquinamento ambientale della catena di fornitura.

Lieb e Lieb, 2012

Weijers et al., 2012

Large et al., 2013

Rossi S. et al., 2013

Studio delle strategie innovative intraprese dai LSP nell'ambito dell'eco-efficienza, know-how e logistica per contribuire a realizzare una Supply Chain eco-efficiente.
Posta sempre più attenzione ai temi ambientali, mentre i driver di scelta sono ancora quelli tradizionali: prezzo, qualità e puntualità nelle consegne.

Individuazione di iniziative per la sostenibilità ambientale e metriche per la misurazione delle prestazioni ambientali dei LSP e dei processi di trasporto.

Nel mercato Europeo rallentamento degli investimenti in tema di sostenibilità. La sostenibilità non è elemento discriminante per attrarre un maggior numero di clienti, l'aspetto economico è il principale driver di scelta. La sostenibilità: fattore di costo più che di efficienza economica.

Circa 1'88\% dei LSP indagati hanno incluso la sostenibilità negli obiettivi aziendali. Affidabilità e costo i principali driver di scelta, la sostenibilità gioca un ruolo minore.

I LSP tedeschi seguono le linee guida del piano di sviluppo sostenibile dell'UE:

- riduzione del trasporto e del CO2;

- riduzione dell'uso del suolo;

- scelta del vettore considerando aspetti sostenibili;

- costante miglioramento delle condizioni di lavoro;

- valorizzazione del lavoro qualificato.

Le principali strategie riguardano: imballaggi, ottimizzazione dei percorsi, educazione dei dipendenti, attività di riciclaggio e conversione dei combustibili. Mancanza di strumenti per implementare e misurare i risultati di politiche eco-efficienti. 
Tab. 4 - Segue

\begin{tabular}{ll}
\hline Autore/Anno & Obiettivo di ricerca \\
\hline Kudla e & $\begin{array}{l}\text { Sostenibilità nelle relazioni diadiche } \\
\text { Klaas-Wissing, 2012 } \\
\text { tra acquirente e LSP, approfondimento } \\
\text { della conoscenza sulle motivazioni } \\
\text { e sulle metodologie del comportamento } \\
\text { sostenibile implementato degli operatori } \\
\text { logistici. }\end{array}$
\end{tabular}

Mass et al., $2014 \quad$ Attività di prevenzione dell'inquinamento ed una gestione responsabile delle capacità aziendali quali strumenti di differenziazione in grado di generare un vantaggio competitivo nel settore dei LSP.

Dey et al., 2011

Mallidis et al., 2012

Lammgard e

Andersson 2014
Sviluppare un modello strategico-tattico di supporto alle decisioni manageriali per valutare l'impatto ambientale legato alle emissioni inquinanti del trasporto .

L'importanza che imprese offerenti servizi di trasporto, attribuiscono agli aspetti ambientali, in particolare alla riduzione delle emissioni di $\mathrm{CO} 2$, con analisi del periodo 2003-2012.

\section{Considerazioni rilevanti}

Differenze significative negli sforzi verso la sostenibilità tra grandi e PMI, rilevazione di problemi di agenzia, inadeguatezza degli stimoli e risposte nelle relazioni.

La prevenzione nell'inquinamento ed una gestione responsabile delle capacità aziendali può effettivamente generare un vantaggio di differenziazione rispetto ad altre imprese.

Riduzione dell'uso improprio delle risorse, in particolare le non rinnovabili e riduzione del $\mathrm{CO} 2$ risulta una necessità etica ed economica che può costituire elemento di differenziazione e generatore di un incremento di valore per il marchio.

La cooperazione tra più imprese nel settore industriale, es. ottimizzazione congiunta dei carichi e condivisione dei magazzini, tramite LSP, può generare una riduzione delle emissioni inquinanti e dei costi.

Qualità ed affidabilità del trasporto, copertura geografica, e basso prezzo riscuotono maggiore attenzione rispetto a temi ambientali.

Fonte: ns. elaborazione.

Dall'analisi della letteratura, nonostante l'operatore logistico emerga come attore importante della filiera produttiva, ancora poco è scritto sul contributo dell'operatore logistico alla sostenibilità della supply chain.

2.2.2. Il possibile contributo degli operatori logistici per la gestione della supply chain sostenibile

Come mostra il paragrafo precedente, gli studi della letteratura esistente sugli operatori logistici e la sostenibilità si riferiscono quasi esclusivamente 
alla sostenibilità ambientale e l'eco-efficienza, mentre manca un approccio sistemico che comprenda contestualmente le tre dimensioni: ambientale, sociale ed economica. Inoltre, il potenziale degli operatori logistici come "abilitatori" della gestione sostenibile della supply chain non è molto dibattuto nelle ricerche sin qui svolte (Kudla e Klaas-Wissing, 2012). L'intento del presente lavoro è proprio di contribuire a colmare questo gap.

Le attività logistiche, e in particolare il trasporto, possono provocare diversi effetti negativi sull'ambiente naturale, come l'inquinamento atmosferico, e sulla sicurezza delle persone, come gli incidenti stradali (Murphy et al., 1994; Rondinelli e Berry, 2000; Wolf e Seuring, 2010; Rossi et al., 2013). In ottica di sostenibilità ambientale, in particolare, tra i diversi settori di servizi quello della logistica può essere più inquinante di tutti gli altri (Skjoett-Larsen, 2000; Wu e Dunn, 1995). I trasporti in generale - nell'ambito delle attività logistiche - costituiscono, infatti, la maggior parte delle emissioni globali (World Economic Forum, 2009); in Europa nel 2009 ha provocato il 30\% di emissioni di $\mathrm{CO} 2$ e il tasso tende ad aumentare in paragone ad altri settori che sono riusciti invece a invertire la tendenza (European Commission, 2012). In ottica di sostenibilità sociale, in particolare, il settore logistico è ad alta intensità di occupati (Kudla e Klaas-Wissing, 2012). Le attività degli operatori logistici hanno rilevanti impatti ambientali e sociali, oltre che economici.

Dey et al. (2011) identificano quattro ragioni per cui è importante che si investa nella sostenibilità delle attività logistiche: il valore del marchio, l'uso improprio delle risorse, l'intervento istituzionale, e gli standard internazionali ed i regolamenti. Ecco, quindi, che con la pressione di una serie di soggetti interessati, compresi i consumatori, gli investitori e i policy maker, la sostenibilità degli attori economici che offrono servizi logistici tende a ricevere sempre più attenzione (Lieb e Lieb, 2010).

La Commissione Europea nel 2001 afferma che l'obiettivo per il settore logistico è quello di "disconnect mobility from its adverse effects". Molte grandi aziende operanti nel settore logistico hanno aumentato il loro impegno in programmi di sostenibilità come fonte di vantaggio competitivo nonostante la recessione (Lieb e Lieb, 2010). Come afferma Prokesch (2010) "adding sustainability into the corporate strategy has become about meeting the expectations of investors while taking into account the long-term impact that operations have on the community and environment". Per di più, come diversi autori rilevano (Wolf e Seauring, 2010; Lammgard e Andersson, 2014), il supporto sostenibile degli operatori logistici ai propri clienti è sempre più spesso rispetto al passato un requisito di selezione del fornitore logistico da parte proprio del cliente business.

Gli operatori logistici hanno potenzialmente la capacità di contribuire ad attenuare $i$ tre aspetti che sono più frequentemente menzionati in letteratura come barriere all'implementazione di supply chain sostenibili, ovvero: (1) i maggiori costi (2) la complessità e il maggior impegno di coordinamento, e (3) l'insufficiente, o addirittura mancante, comunicazione tra attori lungo la 
supply chain (Seuring e Muller, 2008). Questo compito è può essere svolto da quegli operatori logistici capaci non solo di eseguire fisicamente una o più attività logistiche in base alle decisioni tattiche e strategiche assunte dal proprio cliente, ma capaci di acquisire la responsabilità del coordinamento di parti più o meno ampie del processo logistico, sostituendosi ai propri clienti (dell'industria e/o della distribuzione) nell' organizzarle e nell' attuarle, assumendo decisioni con un certo grado di autonomia orientate a (1) ridurre i costi (2) aggregare e quindi semplificare il coordinamento e (3) garantire la necessaria comunicazione lungo la supply chain. Questa capacità può derivare dal ruolo che alcuni operatori sanno ritagliarsi nell' ambito della supply chain in termini di supporto operativo, gestionale e progettuale su tutti gli elementi della rete logistica, ovvero a livello di nodi (o punti), di archi (o segmenti) e, soprattutto, di interfacce (o congiunzioni), poiché in tali punti di contatto vi è, in genere, un passaggio di responsabilità che può creare una discontinuità - fisica, operativa, temporale - (Cozzolino, 2009; Massaroni e Cozzolino, 2012) che può determinare una minore attenzione alle necessità sostenibili.

Anche se l'importanza della sostenibilità degli operatori logistici emerge in alcuni contributi, purtuttavia molto resta ancora da capire a livello empirico in termini di specifiche azioni intraprese da questi attori nell'ambito delle tre dimensioni della sostenibilità. Per un approfondimento in questa direzione basata sulla tripartizione della sostenibilità si è considerata la crescente tendenza delle imprese ad esprimere volontariamente il proprio impegno sostenibile attraverso le attività di "sustainability reporting".

\subsubsection{Il reporting di sostenibilità nel settore della logistica}

Una possibile espressione di maggiore consapevolezza delle imprese verso le questioni ambientali e sociali, si esprime attraverso le attività di sustainability reporting (SR).

Per SR vengono intese le pratiche "of measuring, disclosing, and being accountable to internal and external stakeholders for organizational performance towards the goal of sustainable development" (GRI, 2006, p. 3 ), dove per corporate sustainability report si intendono i "public reports by companies to provide internal and external stakeholders with a picture of the corporate position and activities on economic, environmental and social dimensions" (WBCSD, 2002).

Un crescente numero di imprese che tende a comunicare le azioni svolte a favore della sostenibilità, per esempio attraverso la pubblicazione dei bilanci sociali o dei report di sostenibilità, è guidato da una molteplicità di obiettivi. Da una parte c'è l'intento di voler soddisfare svariati interessi provenienti dagli stakeholders sia interni che esterni all'organizzazione. Dall'altra parte, tenendo conto della volontarietà che distingue queste azioni, il SR mira a realizzare diverse funzioni. Una si potrebbe nominare "conosciti- 
va", in quanto permette di comprendere lo stato attuale delle tre dimensioni cioè economica, ambientale e sociale; l'altra "comunicativa" degli sforzi e progressi raggiunti in tema di sostenibilità (Lozano e Huisingh, 2011). SR si presenta come uno strumento di valutazione d'impresa, ma anche come un'affidabile fonte pubblica di informazioni e conoscenze (Prado-Lorenzo et al. 2009).

Legendre e Coderre (2013) identificano che la decisione di adesione ai determinati standard (es.GRI) sia positivamente correlata alla dimensione e profittabilità aziendale, ma dipende anche dalla rischiosità dell'industria e cultura del business di un dato paese.

Hahn e Kuhnen (2013) elencano una serie di fattori esterni quali l'esposizione mediatica e la pressione dagli stakeholder, ritenendoli rilevanti verso l'elaborazione della rendicontazione di sostenibilità.

Anche se, il SR implica la presentazione da parte di imprese della rendicontazione non solo finanziaria, ma anche quella socio-ambientale, realizzando così la "rendicontazione olistica delle performance aziendali" (Supino e Sica, 2011, pp. 84), esso può rivelarsi uno strumento verso la corporate sustainability (Hahn e Kuchnen, 2013). L'adozione del reporting integrato presenta numerosi vantaggi: l'ottimizzazione nell'assegnazione delle risorse, il miglioramento della gestione del rischio, maggiore precisione nelle informazioni non-finanziarie, il miglioramento dell'immagine (Frias-Aceituno et al., 2014), maggiore ascolto e coinvolgimento degli stakeholder e diminuzione del rischio reputazionale (Golinelli e Volpe, 2012). Feijoo et al. (2014) classificano i comportamenti di imprese appartenenti ai diversi settori verso le problematiche del corporate social reporting in quattro tipi, in base ad una variabile di tipo qualitativo (credibilità) e quantitativo (disclosure): novice "principiante", Cautious "prudenti", Chattering "chiacchierone" e Leading "leader". Il settore della logistica viene riconosciuto come leader caratterizzato da un elevato grado di disclosure e credibilità e quindi elevata anche la sensibilità verso le questioni ambientali.

La rendicontazione delle performance di sostenibilità scelta in questo lavoro si basa sullo standard Global Reporting Initiative, perché esso permette di osservare e misurare tre aspetti della sostenibilità contemporaneamente (Supino e Sica, 2011; Manetti, 2011), ma anche perché è stato riconosciuto da diversi ricercatori come il più diffuso a livello mondiale (Marimon et al., 2012; Roca e Searcy, 2012; Legendre e Coderre, 2013).

\section{Metodologia}

I dati sono stati raccolti on desk utilizzando la banca dati di Global Reporting Initiative (Gri) la quale ha costituito la fonte principale di dati. I siti internet delle singole aziende consultate nelle successive fasi della ricerca hanno costituito la fonte secondaria. 
Cercando di rispondere agli obiettivi del presente lavoro, si è voluto focalizzare l'attenzione sulle piccole, medie e grandi imprese operanti nel settore della logistica in Europa (1). Sono stati inclusi nel set d'indagine i report più recenti al momento dell'avvio della parte empirica di questo progetto di ricerca (al 30 settembre 2014).

Inizialmente, sono stati individuati 21 report. Attraverso l'applicazione dei criteri oggettivi resasi indispensabile ai fini dell'analisi di ogni singolo report, sono state incluse nel set d'indagine i report in lingua inglese pubblicati sul sito GRI o sui siti aziendali.

Per assicurare la completezza di lavoro, si è deciso di analizzare i siti web al fine di ottenere delle informazioni circa la mission, l'attività principale svolta e altri elementi caratterizzanti.

Con il criterio soggettivo si è voluto focalizzare l'attenzione sul trasporto di merci escludendo quello di persone.

Questa selezione ha portato ad avere il set d'indagine completo pari sei aziende operanti nel settore della logistica sul territorio europeo nel settore privato.

È stata condotta la content analysis di tipo qualitativo. La content analysis "has been widely used as it is away to infer from data what would be too costly or too obtrusive to obtain by the use of other techniques" (Krippendorff,1980, p.51). Inoltre, tale tecnica è spesso applicata nello studio e analisi dei rapporti sulla sostenibilità (Delai e Takahashi, 2013).

\section{Risultati}

\subsection{Il quadro generale degli operatori logistici inclusi nel set finale d'inda- gine}

Il quadro generale relativo alle imprese incluse nel set finale d'indagine è rappresentato nella tabella 5; essa è stata costruita in base alle informazioni disponibili sui siti delle aziende $\mathrm{e}$ in base ai report di sostenibilità pubblicati.

Si può notare la notevole presenza delle aziende provenienti dal territorio nord europeo. Un altro fatto potenzialmente rilevante riguarda la longevità di imprese analizzate, di cui tre hanno superato il secolo di attività. I report sulla sostenibilità vengono nominati in modo diverso da diversi soggetti, ma anche il numero di pagine di ogni report è molto varabile. Ciò parzialmente può derivare dal fatto che il prevalente numero di aziende inserisce la parte relativa alla "corporate responsibility" oppure "corporate social responsibility" in report annuali che descrivono i risultai raggiunti che vanno oltre le considerazioni sulla sostenibilità.

1. Il territorio Europeo si rivela ad essere il leader mondiale nell'adozione del GRI dalla sua fondazione. 


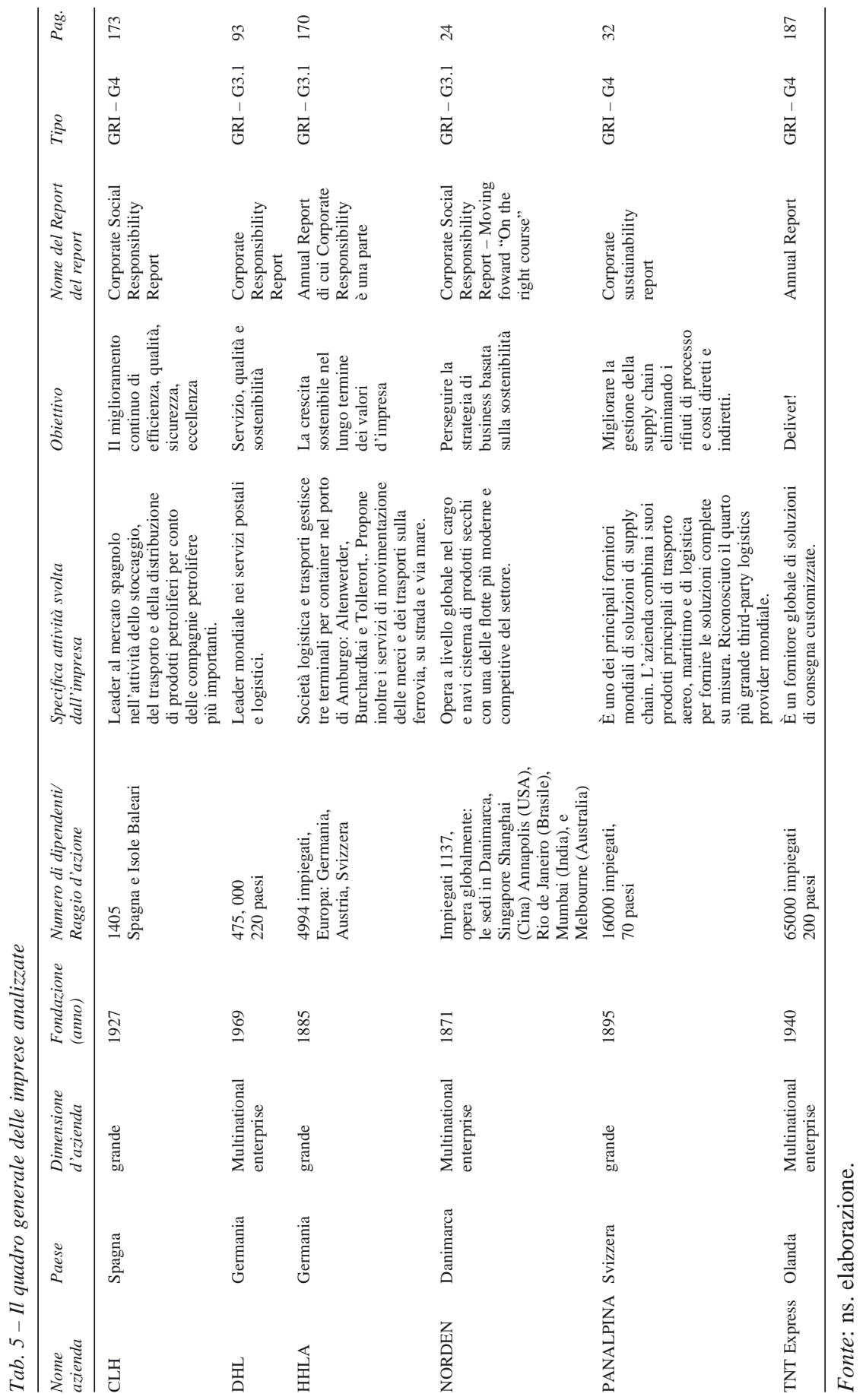




\subsection{Analisi di sostenibilità: iniziative}

Gli indicatori utilizzati sono quelli riportati all'interno del documento denominato Linee guida per il reporting di sostenibilità, reperibile, in lingua italiana, sul sito https://www.globalreporting.org/languages/italian/Pages/default.aspx. Inoltre, è opportuno precisare che il GRI Reporting Framework, con riferimento alle tre dimensioni della sostenibilità (economica, ambientale e sociale), distingue tra indicatori core, utilizzabili universalmente e rilevanti per tutte le imprese, e Additional (facoltativi).

Per la dimensione economica sono stati approfonditi i tre livelli che la caratterizzano; ossia: performance economica, presenza sul mercato ed impatti economici indiretti.

Per quanto riguarda, invece, la dimensione ambientale sono stati analizzati, relativamente alle aziende esaminate, i seguenti aspetti: materie, energia, acqua, biodiversità, emissioni scarichi e rifiuti, prodotti e servizi, conformità (compliance), trasporti, generale.

Considerate le prime due dimensioni, è stato necessario procedere all'analisi della dimensione sociale. In particolare sono stati esaminati i seguenti indicatori di performance: 1) pratiche di lavoro e condizioni di lavoro adeguate (aspetti: occupazione, relazioni industriali, salute e sicurezza sul lavoro, formazione e istruzione e diversità e pari opportunità); 2) diritti umani (aspetti: pratiche di investimento e approvvigionamento, non discriminazione, lavoro minorile, lavoro forzato, pratiche di sicurez$\mathrm{za}$, diritti delle popolazioni indigene, diritti delle popolazioni indigene); 3) società (aspetti: collettività, corruzione, contributi politici - approccio nei confronti di politica/istituzioni, comportamenti anti-collusivi, conformità - compliance); 4) responsabilità di prodotto (aspetti: salute e sicurezza dei consumatori, etichettatura di prodotti e servizi - labeling, marketing e comunicazione, rispetto della privacy, conformità - compliance).

Si riportano di seguito i risultati delle analisi eseguite per la dimensione economica nella tabella 6 , per la dimensione ambientale nella tabella 7 , e per la dimensione sociale nelle tabelle $8,9,10$ e 11 . 


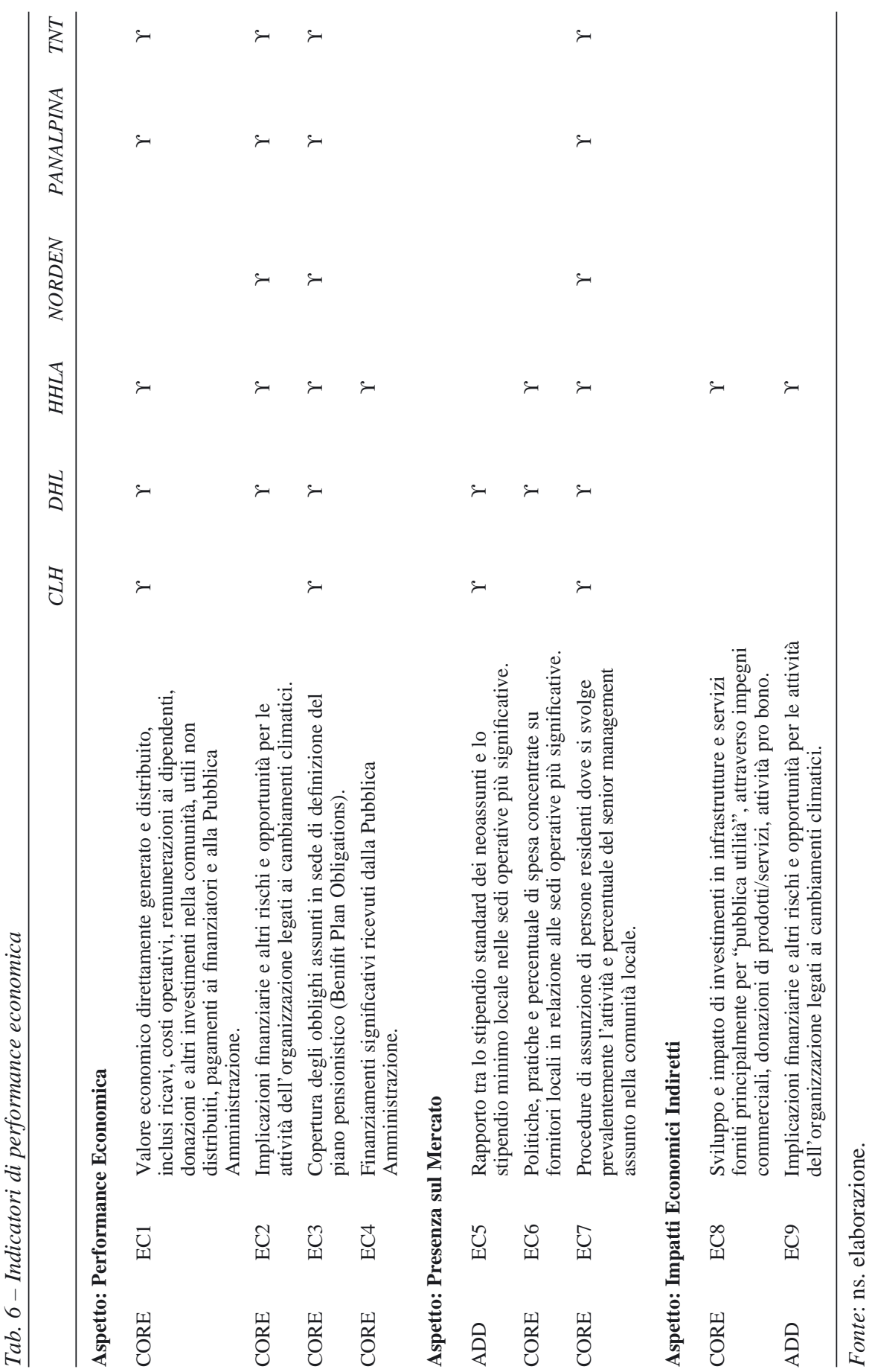




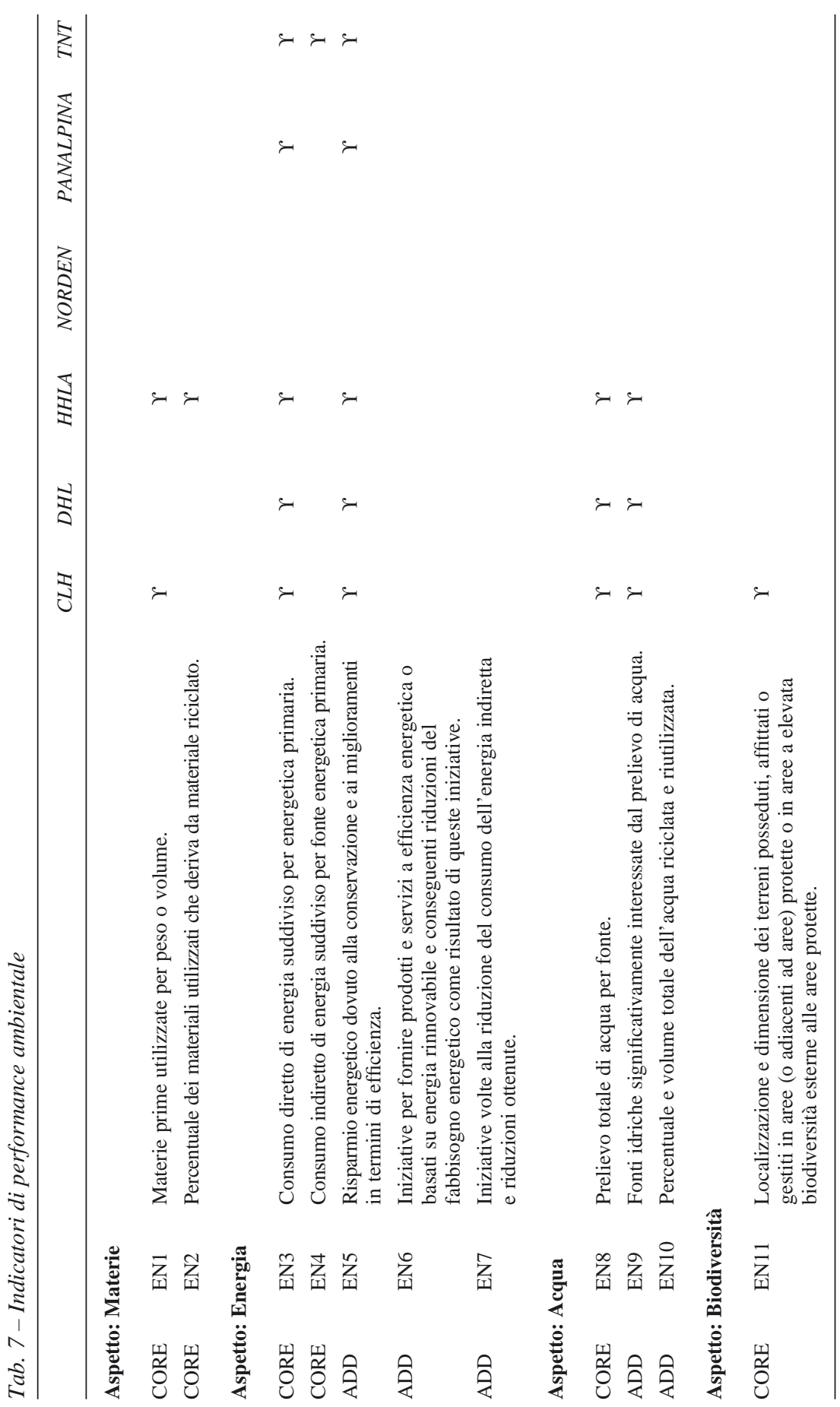
mezzo effettuata e la sua messa a disposizione di terzi, sia in forma gratuita sia a pagamento. 


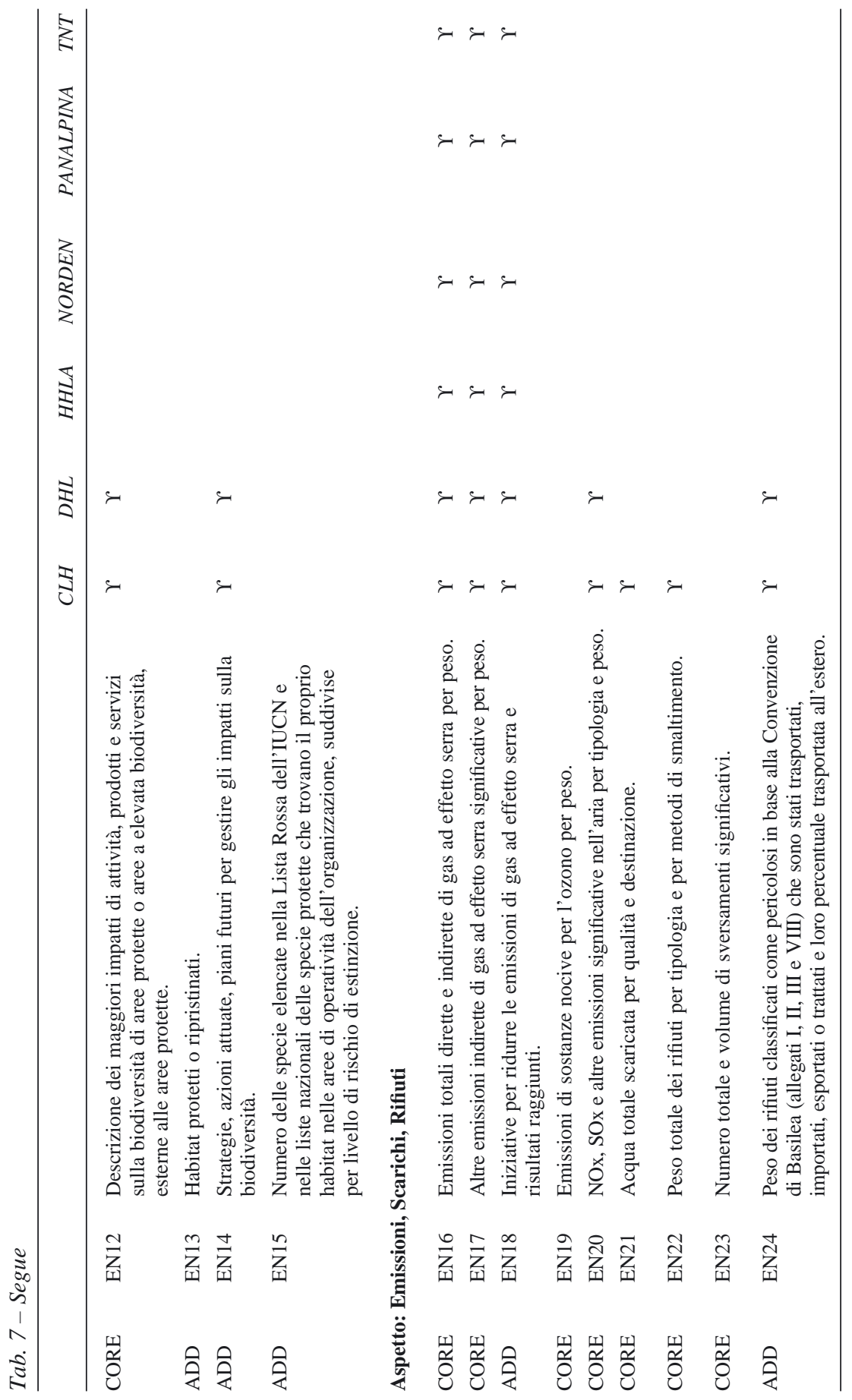




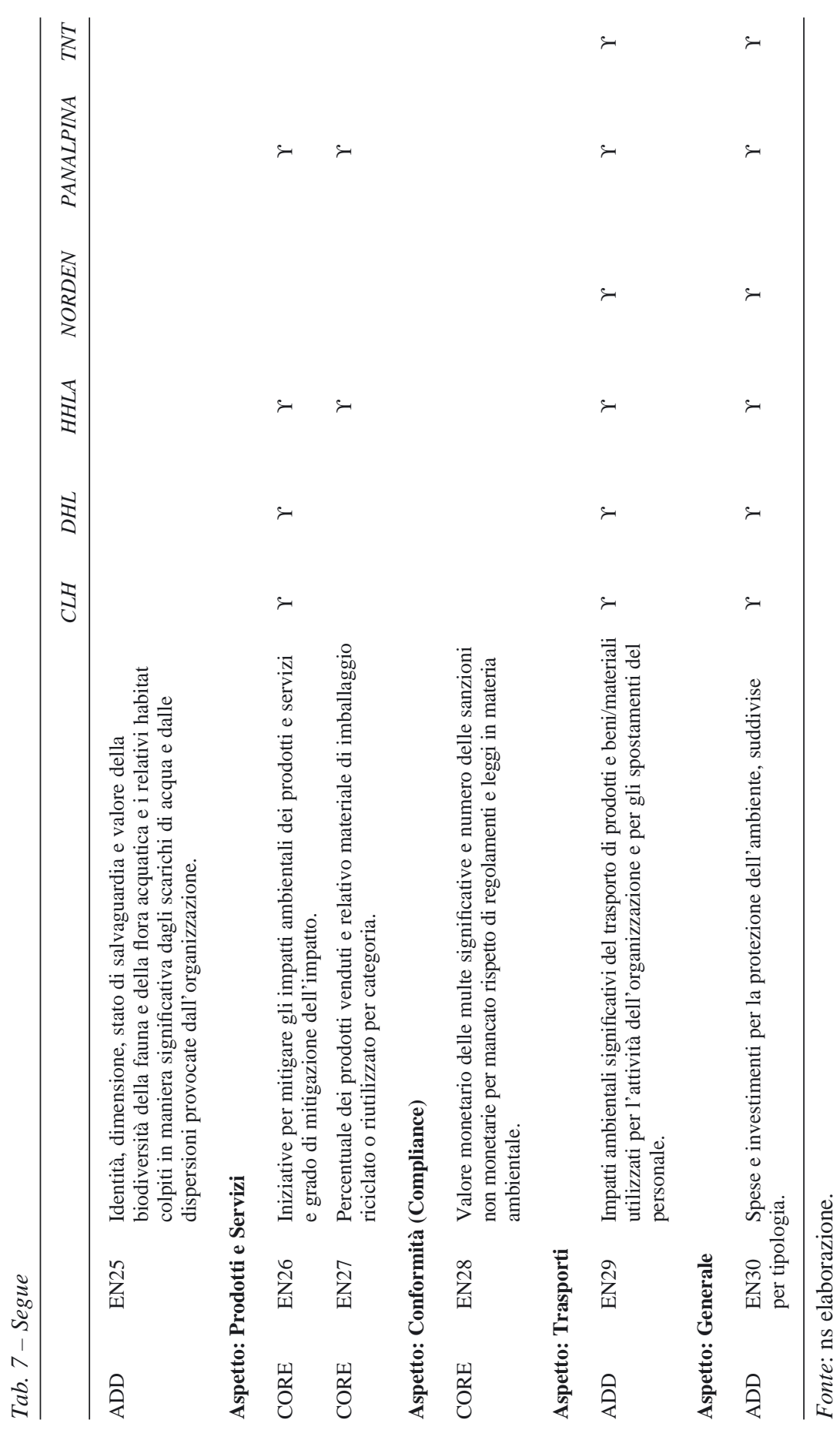
mezzo effettuata e la sua messa a disposizione di terzi, sia in forma gratuita sia a pagamento. 

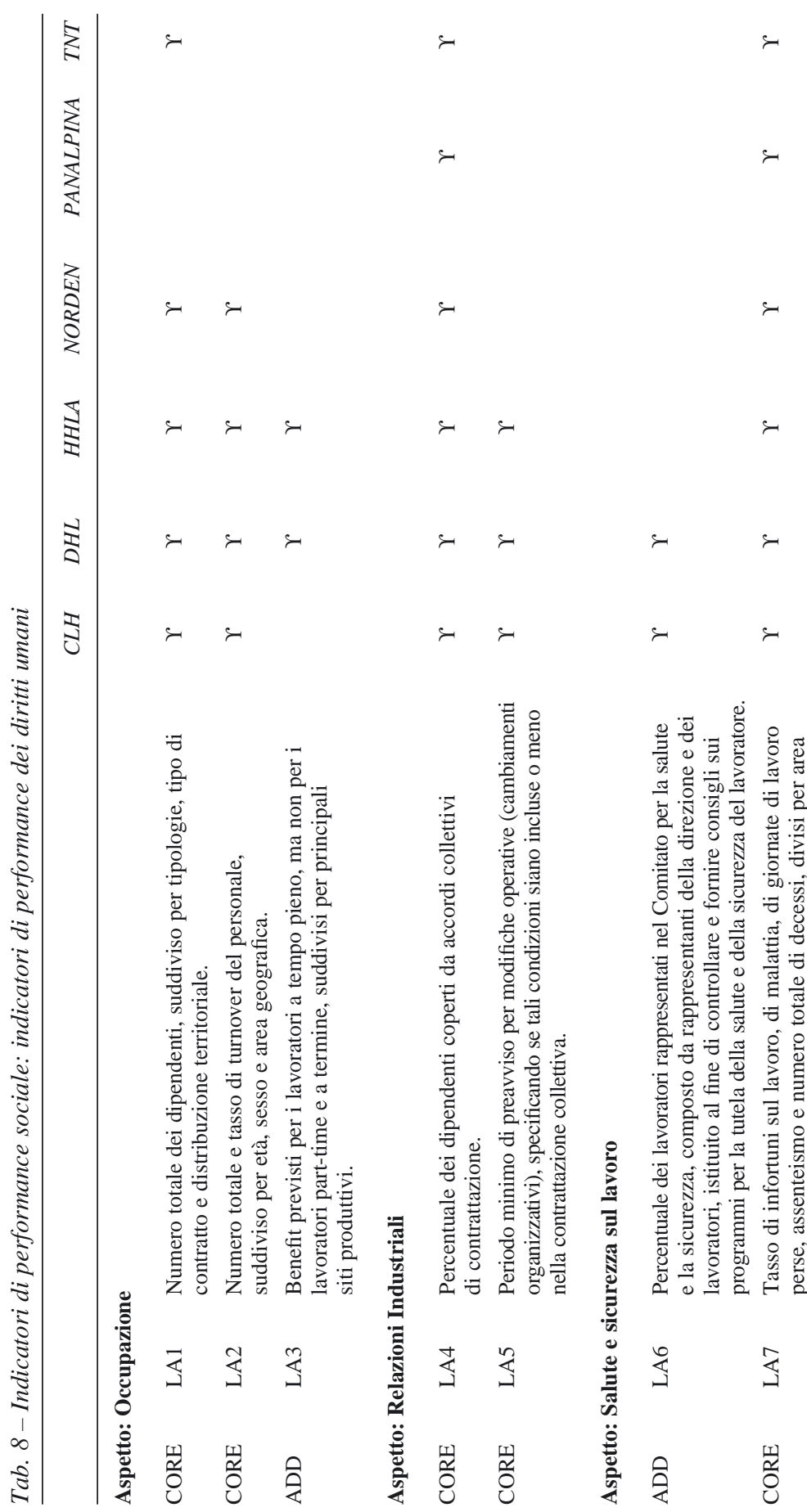

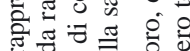

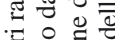
응 준

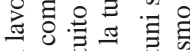

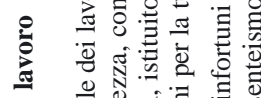

至
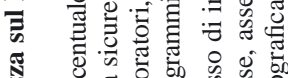

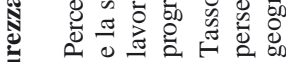

苞芯出

氚

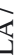

蓄

뙹 mezzo effettuata e la sua messa a disposizione di terzi, sia in forma gratuita sia a pagamento. 


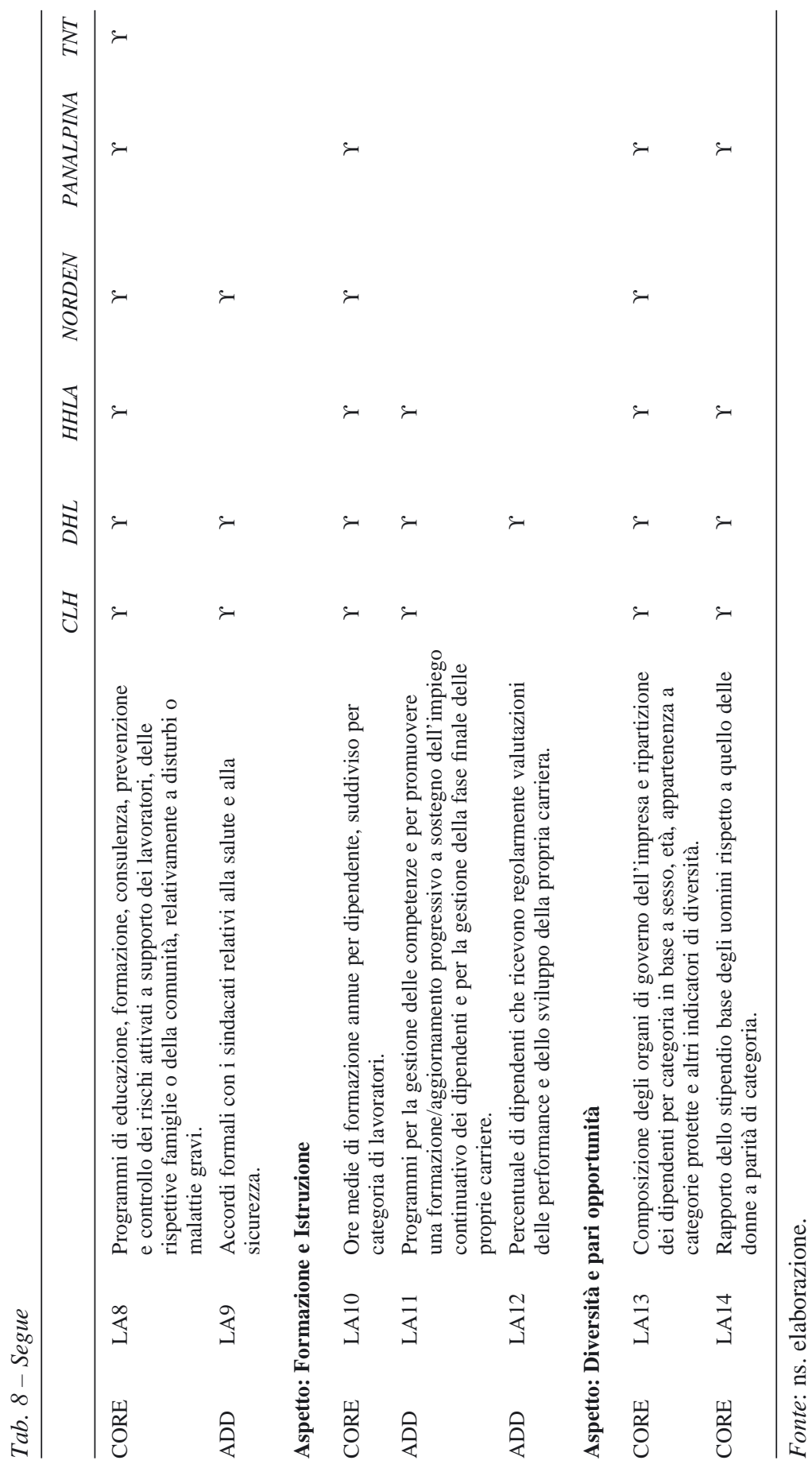

Copyright (C) FrancoAngeli

N.B: Copia ad uso personale. È vietata la riproduzione (totale o parziale) dell'opera con qualsiasi mezzo effettuata e la sua messa a disposizione di terzi, sia in forma gratuita sia a pagamento. 


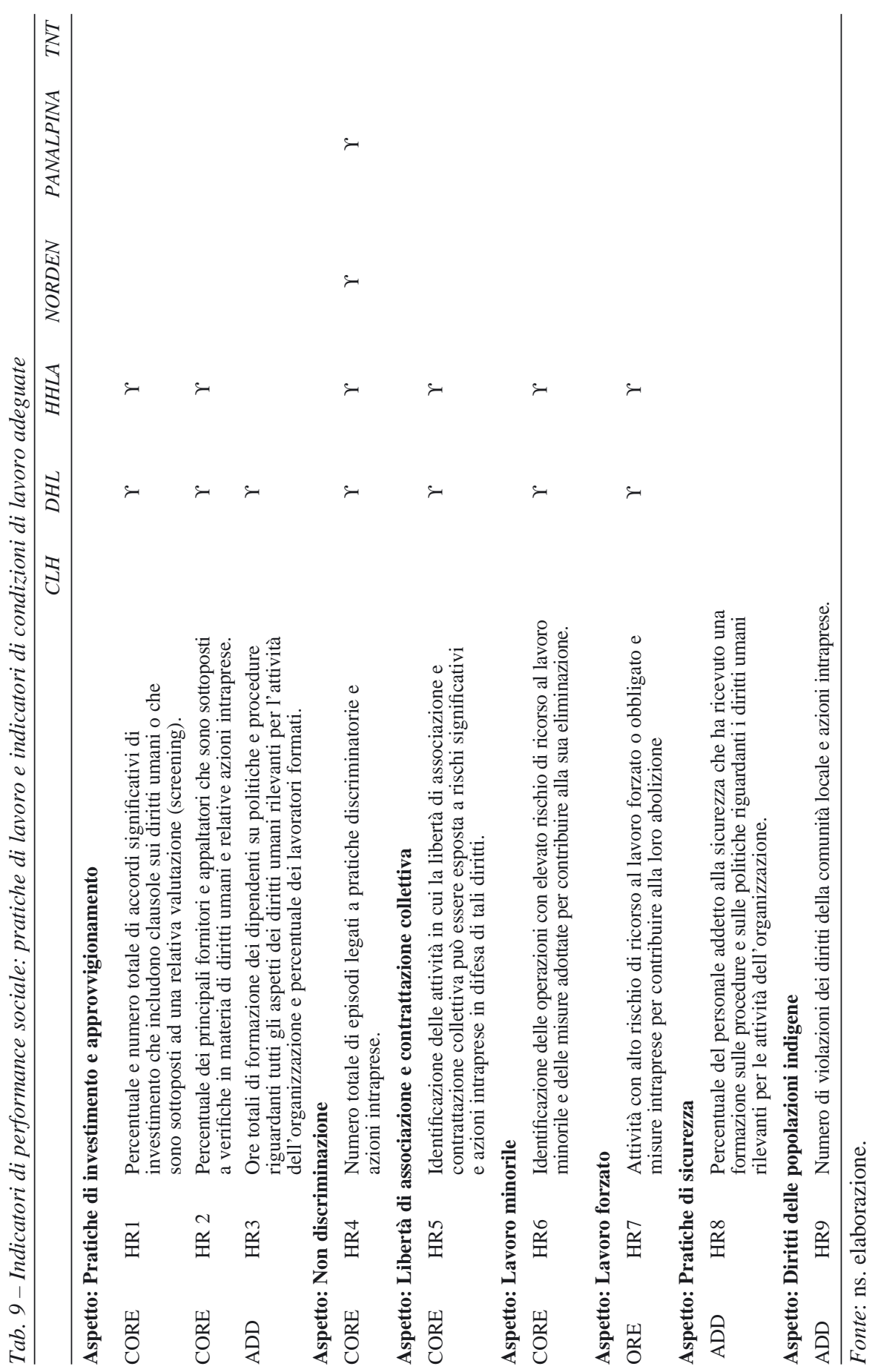




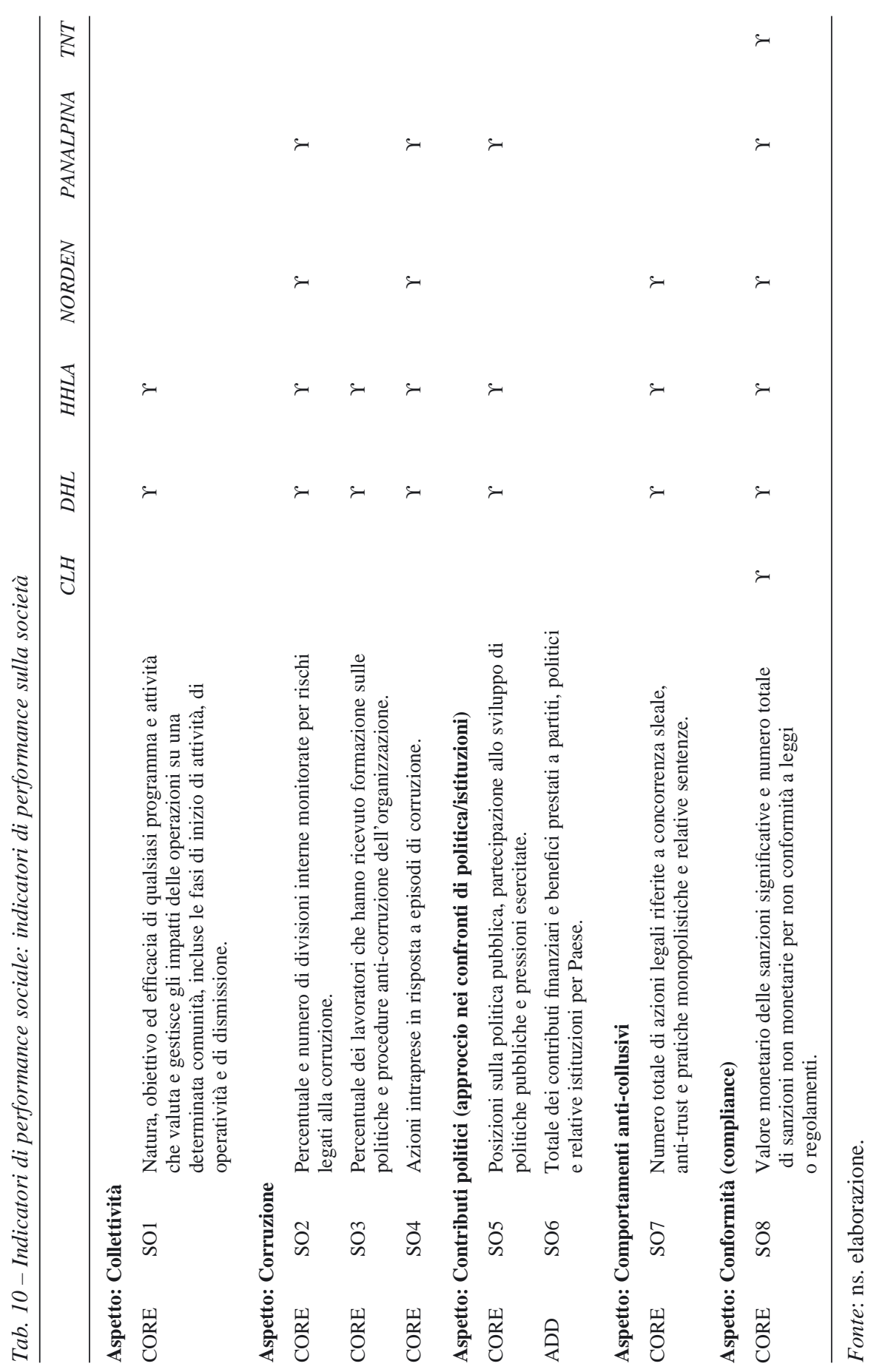




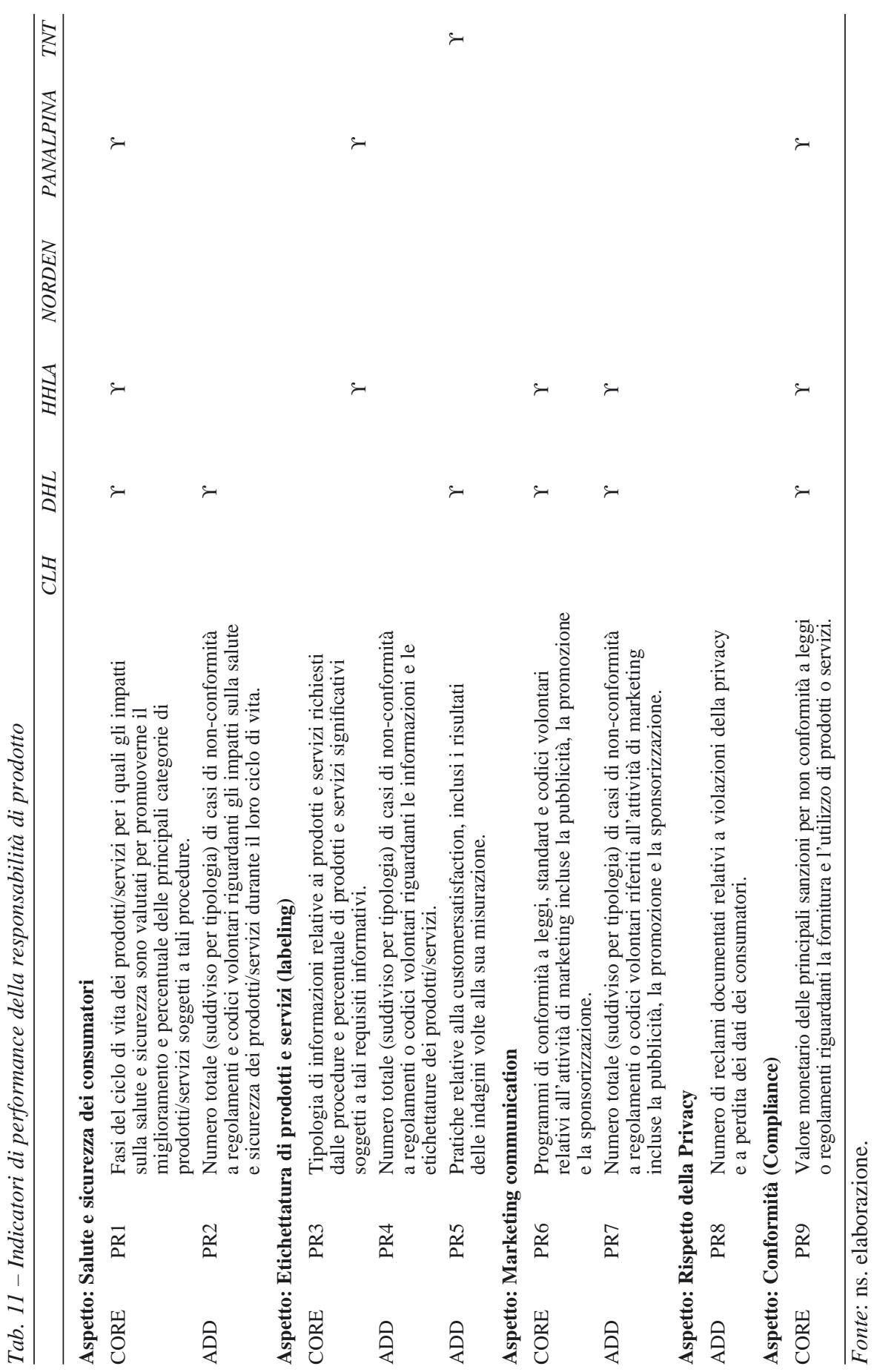




\section{Discussioni}

I risultati di questa indagine empirica mettono ben in evidenza come le imprese di logistica possano contribuire alla sostenibilità in un duplice modo:

- in qualità d'impresa; e,

- in qualità di fornitore di servizi alle altre imprese, industriali e commerciali.

Gli operatori logistici analizzati, infatti, investono in sostenibilità attraverso innovazioni da incorporare a livello di prodotti o di processi per la propria organizzazione; non solo: in virtù della loro vocazione di servizio alle imprese industriali e commerciali, gli operatori logistici possono inoltre "moltiplicare" gli effetti positivi di un loro intervento in senso sostenibile per tutti i propri clienti serviti (Cozzolino, 2009; Massaroni e Cozzolino, 2012).

In qualità di impresa attenta alle tematiche di sostenibilità si possono riportare a titolo di esempio i casi delle seguenti iniziative riscontrate nel reporting analizzato.

CLH nel 2013 consegue il premio "Best Environmental Conduct in Companies", nell'ambito del concorso bandito dall'aeroporto Madrid-Barajas.

DHL, in tema sociale, la strategia aziendale prevede un impegno alle questioni sociali al di là dei confini delle attività espletate: il gruppo attraverso i programmi GoHelp e GoTeach concentra la sua attenzione sulla gestione delle emergenze derivanti dalle catastrofi e sulla pianificazione di adeguate attività formative.

HHLA nel 2011 ha installato un impianto fotovoltaico sul tetto del Container Terminal Tolleror che, grazie anche all'ottimizzazione computerizzata dei container, consente di ridurre il consumo di energia e l'inquinamento.

NORDEN ha adottato uno specifico piano per la gestione delle politiche ambientali; inoltre, al fine di fissare gli obiettivi di riduzione delle emissioni di CO2, la Norden fa riferimento alla International Maritime Organization (IMO) e all'indice operativo di efficienza energetica (EEOI - Energy Efficiency Operational Indicator).

PANALPINA nel 2013 ha sviluppato il progetto PanGreen, un programma globale per lo sviluppo di soluzioni verdi.

TNT ha partecipato a numerosi programmi comunitari di ricerca (CITYLOG, STRAIGHTSOL, FREVUE), che mirano a generare soluzioni a "zero emissione" per le consegne nei centri abitati.

In qualità di fornitore di servizi attento alle tematiche di sostenibilità per i propri clienti si possono riportare a titolo di esempio i casi delle seguenti iniziative riscontrate nel reporting analizzato.

CLH nel 2013 ha conseguito la certificazione UNE-EN ISO 14001, confermando così il proprio impegno alle migliori pratiche ambientali. 
DHL, in ambito di eco-efficienza, ha implementato un programma di protezione ambientale denominato GoGreen, che ha l'intento di valorizzare gli obiettivi ambientali all'interno del Gruppo e aumentare il livello di consapevolezza dei dipendenti aziendali rispetto ai problemi ambientali.

HHLA propone recentemente numerose misure di prevenzione, tra le quali la redazione di apposite linee guida, adottate per gestire il rischio ed evitare pericoli e danni fisici alle risorse umane a vario titolo coinvolte nelle attività aziendali: clienti, fornitori, dipendenti, etc..

NORDEN è molto attenta a garantire che le sue navi siano sicure e gestite rispettando elevati standard qualitativi. L'azienda si propone di garantire, a tutte le persone a vario titolo impegnate nei processi produttivi, un contesto lavorativo attraente, sicuro e sano.

PANALPINA ha implementato un approccio di gestione integrata (Integrated Management System - IMS), conforme alle principali norme internazionali ISO 9001, ISO 14001 e OHSAS 18001, che consente di misurare e migliorare i risultati aziendali.

TNT ha adottato un sistema di gestione ambientale globale per pianificare, implementare, monitorare, gestire e migliorare l'impatto delle sue attività. Per assicurare coerenza e adeguati risultati, prevede che tutte le attività debbano essere conformi alla norma ISO 14001 e le performance vengono misurate attraverso indicatori KPI relativi al risparmio energetico e all'emissioni di CO2. L'azienda è impegnata a mitigare l'impatto aziendale con un focus sulle seguenti tre aree: 1) Operazioni: ridurre le emissioni di CO2, migliorando la qualità dell' aria, attraverso una gestione aziendale efficiente, compresi i subappaltatori; 2) Clienti: fornire informazioni in materia di emissioni di CO2 ai clienti e aiutarli a ridurre le loro emissioni; 3) Persone: incoraggiare i dipendenti e i subappaltatori alla adozione di adeguate modalità operative, sensibilizzandoli alla tutela ambientale.

In riferimento al loro ruolo di fornitori, quindi, essi possono sostenere $\mathrm{i}$ propri clienti business $\mathrm{a}(\mathrm{d})$ :

- allinearsi alle limitazioni ambientali e alle tutele sociali imposte dalla politica a livello locale, nazionale ed internazionale, oppure di anticiparne gli standard;

- incrementare i benefici economici, soprattutto in relazione alla riduzione dei costi;

- sviluppare la strategia di differenziazione basata sull'offerta di prodotti con benefici ecologici e con cura dell'eticità per quei clienti disposti a pagare un premium price.

Rispetto a questo ultimo punto, alcuni studi in letteratura, tra cui quello di Lammgård (2012), pongono in evidenza come gli aspetti di sostenibilità degli operatori logistici non siano necessariamente riconosciuti con il corrispettivo aumento di prezzo, ma siano ritenuti dai clienti business un elemento compreso nel prezzo del servizio di base. In particolare, alcune evi- 
denze empiriche emerse dalle ricerche di Rossi et al. (2013) attestano che "the quality of the services expected by the customer remains the same. Moreover, they are not willing to pay a premium price for more eco-efficient logistics services"; insomma, in questi casi "customers are still much more cost focused" (Lieb e Lieb, 2010).

\section{Limiti e future linee di ricerca}

Il presente studio evidenzia una crescente propensione e proattività dei LSP verso le sfide dello sviluppo sostenibile, attitudini che si tramutano in vantaggio competitivo. Il ruolo dei LSP grazie alla loro presenza sia a monte che a valle della supply chain impatta fortemente sulla performance sostenibile dell'intera filiera. In quest'ottica diventano di vitale importanza le scelte dei partner business che dovrebbero basarsi sui criteri che rispecchino anche gli interessi ambientali e sociali.

I risultati di questo studio possono essere ulteriormente arricchiti attraverso l'analisi dei report prodotti in un arco di tempo più lungo al fine di evidenziare eventuali cambiamenti intercorsi. Inoltre, può risultare interessante ampliare l'indagine considerando aree geografiche più vaste, oltre al territorio europeo; ciò permetterebbe di cogliere differenze oppure somiglianze nell' agire degli operatori logistici a livello mondiale. Infine, potrebbe rivelarsi utile comprendere ulteriori soggetti nel campione d'indagine che non siano solo operatori logistici, ma anche operatori postali, operatori del trasporto di persone oppure complesse strutture logistiche.

\section{Riferimenti bibliografici}

Ahi, P., Searcy, C., A comparative literature analysis of definitions for green and sustainable supply chain management, in «Journal of Cleaner Production», 52, 2013, pp. 329-341.

Antonnen, M., Halme, M., Houbeckers, E., Nurkka, J., The other side of sustainable innovation: is there a demand for innovative services?, in «Journal of Cleaner Production», 45, 2013, pp. 89-103.

Burritt, R., Schaltegger, S., Accounting towards sustainability in production and supply chains, in «The British Accounting Review», 46, 2014, pp. 327-343.

Carter, C.R., Rogers, D.S., A framework of sustainable supply chain management: moving toward new theory, in «International Journal of Physical Distribution \& Logistics», 38, 5, 2008, pp. 360-387.

Ciliberti, F., Potrandolfo, P., Scozzi, B., Investigating corporate social responsibility in supply chains: a SME perspective, in «Journal of Cleaner Production», 16, 15, 2008, pp. 1579-1588.

Closs, D., Speier, C., Meacham, N., Sustainability to support end-to-end value chains: The role of supply chain management, in «Journal of the Academy of Marketing Science», 39, 1, 2011, pp. 101-116. 
Colicchia, C., Marchet, G., Melacini, M., Proietti, S., Building environmental sustainability: empirical evidence from Logistics Services Providers, in «Journal of Cleaner Production», 59, 2013, pp. 197-209.

Cozzolino, A., Operatori logistici. Contesto evolutivo, assetti competitivi e criticità emergenti nella supply chain, Padova, Cedam, 2009.

Delai, I., Takahashi, S., Corporate sustainability in emerging markets: insights from the practices reported by the Brazilian retailers, in «Journal of Cleaner Production», 47, 2013, pp. 211-221.

Dey, A., Laguardia, P., Srinivasan, M., Building sustainability in logistics operations: a research agenda, Management Research Review, 34, 11, 2011, pp. 1237-1259.

European Commission, EU transport in figures: Statistical pocketbook 2012, Luxembourg, European Union, 2012.

Facanha, C., Horvath, A. (2005), Environmental Assessment of Logistics Outsourcing, in «Journal of Management in Engineering», 21, 1, pp. 27-37.

Fernandez-Feijoo, B., Romero, S., Ruiz, S., Commitment to Corporate social responsibility measured through global reporting initiative reporting: factors affecting the behavior of companies, in «Journal of Cleaner Production», 81, 2014, pp. 244-254.

Font, X., Tapper, R., Schwartz, K., Kornilaki, M., Sustainable supply chain management in tourism, in «Business Strategy and the Environment», 2008, pp. 260 e 271.

Frias-Aceituno, J.V., Rodríguez-Ariza, L., \& Garcia-Sánchez, I.M., Explanatory Factors of Integrated Sustainability and Financial Reporting, in «Business Strategy \& the Environment», 23, 1, 2014, pp. 56-72.

Giunipero, L., Hooker, R., \& Denslow, D., Purchasing and supply management sustainability: Drivers and barriers, in «Journal of Purchasing \& Supply Management», 18, 4, 2012, pp. 258-269

Golinelli, G., Volpe, L., Consonanza, valore, sostenibilità: verso l'impresa sostenibile, Padova, Cedam, 2012.

Govindan, K., Khodaverdi, R., Jafarian, A., A fuzzy multi criteria approach for measuring sustainability performance of a supplier based on triple bottom line approach, in «Journal of Supply Chain Management», 49, 2, 2013, pp. 78-95.

Gri, Sustainability Reporting Guidelines, Version 3. Global Reporting Initiative, Amsterdam, 2006.

Haake, H., Seuring, S., Sustainable procurement of minor items e exploring limits to sustainability, Sustainable Development, 2009, pp. 284 e 294.

Hahn, R., Kühnen, M., Determinants of sustainability reporting: a review of results, trends, theory, and opportunities in an expanding field of research, in «Journal of Cleaner Production», 59, 2013, pp. 5-21.

Hart, S.L., Milstein, M.B., Creating sustainable value. Academy of Management Executive, 17, 2, 2013, pp. 56-67.

Hassini, E., Surti, C., Searcy, C., A literature review and a case study of sustainable supply chains with a focus on metrics, in «International Journal of Production Economics», 140, 1, 2012, pp. 69-82.

Krippendorf, K., Content analysis: An introduction to its methodology, Beverly Hills, CA, Sage, 1980.

Kudla, N.L., Klaas-Wissing, T., Sustainability in shipper-logistics service provider relationships: A tentative taxonomy based on agency theory and stimulus-response analysis, in «Journal of Purchasing \& Supply Management», 18, 4, 2012, pp. 218231. 
Lammgård, C., Intermodal train services: A business challenge and a measure for decarbonisation for logistics service providers, in «Research in Transportation Business \& Management», 5, 2012, pp. 48-56.

Lammgård, C., Andersson, D., Environmental considerations and trade-offs in purchasing of transportation services, in «Research in Transportation Business \& Management», 40, 2014, pp. 45-52.

Large, R.O., Kramer N., Hartmann R.K., Procurement of logistics services and sustainable development in Europe: Fields of activity and empirical results, in «Journal of Purchasing \& Supply Management», 19, 3, 2013, pp. 122-133.

Lee, K.H., Wu, Y., Integrating sustainability performance measurement into logistics and supply networks: A multi-methodological approach, in «The British Accounting Review», 46, 4, 2014, pp. 361-378.

Legendre, S., Coderre, F., Determinants of GRI G3 Application Levels: The Case of the Fortune Global 500, in «Corporate Social Responsibility \& Environmental Management», 20, 3, 2013, pp. 182-192

Lieb, K.J., Lieb, R.C., Environmental sustainability in the third-party logistics (3PL) industry, in «International Journal of Physical Distribution \& Logistics Management», 40, 7, 2010, pp. 524-533.

Lieb, K.J., Lieb, R.C., The European Third Party Logistics Industry in 2011: The Provider CEO Perspective, in «Supply Chain Forum: International Journal», 13, 1, 2012, pp. 2-8.

Linton, J.D., Klassen, R., Jayaraman, V., Sustainable supply chains: An introduction, in «Journal of Operations Management», 25, 6, 2007, pp. 1075-1082.

Lozano, R., Huisingh, D., Inter-linking issues and dimensions in sustainability reporting, in «Journal of Cleaner Production», 9, 2011, pp. 99-107.

Maas, S., Schuster, T., Hartmann, E., Pollution Prevention and Service Stewardship Strategies in the Third-Party Logistics Industry: Effects on Firm Differentiation and the Moderating Role of Environmental Communication, in «Business Strategy \& the Environment», 23, 1, 2014, pp. 38-55.

Mallidis, I., Dekker, R., Vlachos, D., The impact of greening on supply chain design and cost: a case for a developing region, in «Journal of Transport Geography», 22, 2012, pp. 118-128.

Manetti, G., The quality of stakeholder Engagement in sustainability reporting: empirical evidence and critical points, in «Corporate Social Responsibility and Environmental Management», 18, 2011, pp. 110-122.

Marimon, F., Alonso-Almeida, M.M., Rodríguez, M.P., \& Cortez Alejandro, K.A., The worldwide diffusion of the global reporting initiative: what is the point?, in «Journal of Cleaner Production», 33, 2012, pp. 132-144

Massaroni, E., Cozzolino, A., Modelli della produzione industriale, Padova, Cedam, 2012.

Murphy, P.R., Poist, R.F. \& Braunschweig C.D., Management of environmental issues in logistics: current status and future potential, in «Transportation Journal», 34, 1, 1994, pp. 48-56.

Pagell, M., Wu, Z., Building a more complete theory of sustainable supply chain management using case studies of 10 exemplars, in «Journal of Supply Chain Management», 45, 2, 2009, pp. 37-56.

Prado-Lorenzo, J.M., Gallego-Alvarez, I., Garcia-Sanchez, I.M., Stakeholder engagement and corporate social responsibility reporting: the ownership structure effect, in «Corporate Social Responsibility and Environmental Management», 16, 2009, pp. 94-107. 
Prokesch, S., The sustainable supply chain, in «Harvard Business Review», 88, 10, 2010, pp. 70-2.

Rao, P., Holt, D., Do green supply chains lead to competitiveness and economic performance?, in «International Journal of Operations and Production Management», 25, 9, 2005, pp. 898-916.

Roca, L.C., Searcy, C., An analysis of indicators disclosed in corporate sustainability reports, in «Journal of cleaner production», 20, 2012, pp. 103 e 118.

Rondinelli, D., Berry, M., Multimodal transportation, logistics, and the environment: managing interactions in a global economy, in «European Management Journal», 18, 4, 2000, pp. 398-410.

Rossi, S., Colicchia C., Cozzolino, A., \& Christopher, M., The logistics service providers in eco-efficiency innovation: An empirical study, in «Supply Chain Management: An International Journal», 18 6, 2013, pp. 583-603.

Seuring, S., Sarkis, J., Müller, M. and Rao, P., Sustainability and Supply Chain Management: An Introduction to the Special Issue, in «Journal of Cleaner Production», 16, 15, 2008, pp. 1545-1551.

Seuring, S., Muller, M., From a literature review to a conceptual framework for sustainable supply chain management, in «Journal of Cleaner Production», 16, 15, 2008, pp. 1699-1710.

Seuring, S., A review of modeling approaches for sustainable supply chain management, in «Decision support systems», 54, 4, 2013, pp. 1513-1520.

Skjoett-Larsen, T., European logistics beyond 2000, in «International Journal of Physical Distribution and Logistics Management», 30, 5, 2000, pp. 377-387.

Srivastava, S.K., Green supply-chain management: a state-of-the-art literature review, in «International Journal of Management Reviews», 9, 1, 2007, pp. 53-80.

Supino, S., Sica, D., Nuovi paradigmi di rendicontazione d'impresa: il report integrato, in «Esperienze d'impresa», 2, 2011, pp. 81-91.,

Van Hoof, B., Thiell, M., Collaboration capacity for sustainable supply chain management: small and medium-sized enterprises in Mexico, in «Journal of Cleaner Production», 67, 2014, pp. 239-248.

Weijers, S., Glöckner, H.H., Pieters, R., Logistic Service Providers and Sustainable Physical Distribution, in «LogForum», 8, 2, 2012, pp. 157-165.

Winter, M., Knemeyer, A.M., Exploring the integration of sustainability and supply chain management Current state and opportunities for future inquiry, in «International Journal of Physical Distribution \& Logistics Management», 43, 1, 2013, pp. 18-38.

Wolf, C., Seuring, S., Environmental impacts as buying criteria for third party logistical services, in «International Journal of Physical Distribution \& Logistics Management», 40, 1, 2010, pp. 84-102.

World Business Council For Sustainable Development, Sustainable development reporting: Striking the balance. Geneva: World Business Council for Sustainable Development.

WORLD ECONOMIC FORUM (2009), Supply chain decarbonization: The role of logistics and transportation in reducing supply chain carbon emissions, Geneva, World Economic Forum, 2012.

Wu, H.J., Dunn, S.C., Environmentally responsible logistics systems, in «International Journal of Physical Distribution \& Logistics Management», 25, 2, 1995, pp. 20-28. 